Cita bibliográfica: Cruz Coria, E., Olmos Martínez, E. y Ibarra Michel, J.P. (2022). La integración de emprendedores turísticos a las redes de cooperación interempresarial en entornos rurales: Un análisis relacional. Investigaciones Turísticas (23), pp.73-93. https://doi.org/10.14198/INTURI2022.23.4

\title{
La integración de emprendedores turísticos a redes de cooperación interempresarial en entornos rurales: Un análisis relacional
}

The integration of tourism entrepreneurs into inter-firm cooperation networks in rural environments: A relational analysis

Erika Cruz Coria (iD, Universidad Autónoma de Occidente, México ecoria84@hotmail.com

Elizabeth Olmos Martínez (iD, Universidad Autónoma de Occidente, México eolmosm.udo@gmail.com

Juan Pedro Ibarra Michel (iD, Universidad Autónoma de Occidente, México pedro.ibarra@udo.mx

\section{RESUMEN}

Las micro y pequeñas empresas turísticas y los emprendimientos comunitarios en el ámbito rural tienen que hacer frente a diversos desafíos impuestos por el entorno, además de encontrar estrategias para configurar destinos más innovadores. Las redes de cooperación inter-empresarial $(\mathrm{RCl})$ son estructuras de relaciones sociales que les permiten aprovechar eficientemente los recursos locales y activar las capacidades empresariales. Se presenta una revisión de literatura que busca identificar los factores que determinan la integración social (IS) de los emprendedores turísticos en $\mathrm{RCl}$ en entornos rurales; simultáneamente se abordan las ventajas que estos factores generan para su participación en relaciones de cooperación. Es una investigación exploratoria y descriptiva que presenta la revisión de 92 artículos científicos de revistas internacionales. Se optó por un diseño articulado al Análisis Temático (AT) mediante el cual se crearon 41 subcategorías que corresponden a los factores y las ventajas de IS. Para el análisis de la relación entre estos factores se utilizó el software Atlas. ti y, Gephi para visualizar las relaciones y las comunidades de códigos a través de una red. Entre los factores determinantes están los fuertes lazos locales y los lazos con actores externos. Los primeros, influyen en la integración de los emprendedores a redes de cooperación local, mientras que los segundos en su integración en redes para la innovación.

Palabras clave: Integración social; emprendedores; turismo; cooperación inter-empresarial; redes. 


\section{ABSTRACT}

Micro and small enterprises (MSEs) and community-based entrepreneurial projects in rural areas face several challenges imposed by the environment. Furthermore, they must find a way to configure more innovative destinations. Inter-firm cooperation networks (ICN) are structures of social relations that allow them to take advantage of local resources and activate business capacities. This paper provides a literature review in order to identify the factors that determine the social embeddedness (SE) of tourism entrepreneurs in ICN in rural environments. In addition, the advantages that these factors generate for their involvement in cooperative relations are reported. This research is exploratory and descriptive, comprising the review of ninety-two scientific papers. A methodological design based on Thematic Analysis (TA) was selected. Forty-one subcategories were created from the data, which correspond to the factors and advantages of SE. The Atlas. ti software was used to analyse the relations between subcategories and Gephi software was used to visualise relations and code communities through a network. Among the determining factors are strong local ties and ties with external actors.

Keywords: Social embeddedness; entrepreneurs; tourism; cooperation; networks.

\section{INTRODUCCIÓN}

El turismo en el ámbito rural es considerado, en países desarrollados y en vías de desarrollo, como una estrategia para dinamizar las economías locales debido su impacto en términos de valor agregado y en la generación de empleo. Este dinamismo económico se debe, en parte, a la fuerte presencia de micro y pequeñas empresas turísticas (Mypes) y emprendimientos comunitarios (Getz yPetersen 2005; Czernek y Czakon, 2016; Booyens y Rogerson, 2017).

Sin bien, los emprendedores deben hacer frente a una amplia diversidad de desafíos impuestos por las propias características del contexto rural -la creciente globalización, la lejanía con los mercados emisores de turismo, la rivalidad entre destinos turísticos, la escases de mano de obra calificada y un menor desarrollo tecnológico, entre otros - (Farrell et al., 2010); también deben encontrar la forma de configurar destinos más innovadores no sólo para atraer flujos turísticos sino para garantizar el impacto de la actividad en el desarrollo local (Hjalager, 2002, 2010). Esto no sólo depende de sus esfuerzos individuales sino de las estructuras sociales que les permiten aprovechar eficientemente los recursos locales, movilizar las ideas, activar las capacidades empresariales y emprender dinámicas económicas capaces de crear relaciones simbióticas entre las organizaciones y el entorno local (Tolstad, 2014; Seelos et al., 2015).

Para algunos autores, las redes de cooperación inter-empresarial $(\mathrm{RCl})$ constituyen esos recursos valiosos para la generación de valor agregado en el turismo, sin embargo no deben ser vistas únicamente como alianzas comerciales. De acuerdo con Johannisson et al. (2002), las redes constituyen formas de cooperación voluntaria que implican el intercambio de información, aprendizaje mutuo, reciprocidad y comunicación, además de que son mecanismos de control social. Este trabajo de investigación retoma el concepto de integración social (social embeddedness en inglés), el cual permite indagar sobre los factores en la estructura de las relaciones sociales que alientan a los emprendedores a intercambiar información, ideas, valores y diferentes recursos con otras empresas para conformar $\mathrm{RCl}$. Este 
trabajo presenta una revisión sistemática de literatura que pretende identificar los factores que determinan la integración social (IS) de los emprendedores turísticos en $\mathrm{RCl}$ en entornos rurales; simultáneamente se indaga en las ventajas que estos factores generan para su participación en relaciones de cooperación.

En la primera sección se abordan los diferentes enfoques bajo los cuales han sido estudiadas las redes en contextos turísticos. Posteriormente, se expone la importancia de la incorporación de los emprendedores turísticos en $\mathrm{RCl}$ en entornos rurales. En la siguiente sección, se presenta la medodología a través de la cual fueron identificados los factores que determinan la integración social (IS) de los emprendedores turísticos en $\mathrm{RCl}$ y las ventajas de su participación en relaciones de cooperación. Finalmente, se desarrollan los resultados de la investigación y las conclusiones.

\section{LOS ENFOQUES DE REDES EN EL TURISMO}

En la actividad turística son diversos los actores sociales que directa e indirectamente están involucrados en el suministro de servicios, infraestructura, información, entre otros recursos. La cooperación entre los actores es un prerrequisito para el funcionamiento de los destinos en un entorno cambiante y competitivo. Sin importar la escala o las modalidades de turismo que estructuran los destinos, la sinergia social se coloca como una poderosa maquinaria que impulsa el desarrollo sustentable en la actividad (Beritelli, 2011; van der Zee y Vanneste, 2015).

La cooperación entre los actores turísticos puede adoptar la forma de alianzas estratégicas, coaliciones, acuerdos colaborativos u otras formas de cooperación, pero todas son operables a través de redes de relaciones (Albrecht, 2013). Para Hall $(2005,2011)$, una red es un acuerdo de cooperación y colaboración entre organizaciones. Los primeros estudios sobre redes en el turismo se remontan a mediados de los años setenta, cuando Gunn (1977) advertía que la falta de colaboración entre los actores públicos y privados impedía el desarrollo sustentable del turismo. Fue hasta la década de los noventa cuando la temática comenzó a cobrar mayor relevancia (Czernek, 2020).

Son variados los enfoques desde los cuales se ha tratado de descubrir el potencial de las redes en el turismo, para este trabajo de investigación se identifican dos importantes. En el primero, se considera que las redes son necesarias para gestionar las relaciones entre los actores públicos-privados, tanto en el ámbito de la política como de la planificación turística. Desde la perspectiva de la política turística, los trabajos que conforman esta vertiente atribuyen suma importancia a los actores públicos en la planificación del turismo y en la formulación de políticas públicas a diferentes escalas (local, regional, nacional e internacional) (Hall, 2011b; Dredge, 2006; Beaumont y Dredge, 2010; Erkuş y Eraydin, 2010). Esta postura sugiere que el Estado debe limitarse a la coordinación de los actores económicos y sociales, aunque también debe adoptar un papel activo en las redes de turismo (Melián y García, 2003) mediante la generación de alianzas cooperativas y asociaciones con los sectores privados y voluntarios (Jamal y Getz, 1995; Thomas y Thomas, 1999).

En esta vertiente, también son considerados los estudios de redes de las partes interesadas (stakeholders theory) (Hazra et al., 2014; Stoddarta et al., 2019). Para este enfoque, la satisfacción de las necesidades y demandas turísticas deben ser el resultado de la acción conjunta, complementaria y competitiva entre el gobierno, el sector privado y la 
sociedad civil. A escala local, esta propuesta supone interacciones de trabajo cercanas entre los agentes económicos y sociales para promover eficientemente el turismo y el desarrollo local (McCabeet al., 2012; McComb y Boyd, 2016).

En la segunda vertiente, las redes son el marco a través de cual se analizan las relaciones entre operadores turísticos (microempresarios, empresarios, emprendedores, otros) para la innovación, el intercambio de conocimientos, la competitividad y el desarrollo económico. Algunos estudios centran los esfuerzos analíticos en los resultados generados por los emprendedores y empresarios en redes de colaboración (Cátia y Franco, 2016; Novelli et al., 2006). Otros se enfocan en el aprendizaje interorganizacional y en el intercambio de conocimientos entre los empresarios, operadores turísticos y emprendedores como estrategia para la innovación de productos, servicios y destinos turísticos (Keeble, et al., 1999; Booyens y Rogerson, 2016). Esta vertiente ofrece la posibilidad de explorar otros temas que atraviesan el entendimiento de la cooperación inter-empresarial, tales como la confianza, los conflictos de intereses, el liderazgo (Czernek y Czakon, 2016; Kelliher et al., 2018).

Finalmente se observan aquellos trabajos que -desde una perspectiva socioorganizacional - reconocen los problemas complejos que enfrentan las empresas, mismos que van más allá de sus capacidades individuales; por tanto, plantean la resolución de problemas en condiciones de interdependencia con otras (Jamal y Getz, 1995; Trist, 1977). Los estudios que cultivan esta vertiente enfatizan en que la conformación de redes horizontales, no jerárquicas, autorganizadas y de lazos locales densos y débiles pueden derivar en beneficios cuantitativos y cualitativos para las empresas que participan de la red (Booyens y Rogerson, 2016; Brandão et al., 2019). En los últimos años, esta vertiente ha tomado importancia debido a que muestra capacidad explicativa sobre el desempeño de las Mypes y empresas sociales en el ámbito local (Czernek, 2020; Jack y Anderson, 2002; Seelos et al., 2015). Este enfoque permite evidenciar que el desempeño y el comportamiento económico de las empresas turísticas es facilitado por los vínculos que mantienen con otras; la integración social (IS) de los emprendedores en $\mathrm{RCl}$ es visto como un sistema de oportunidades que les brinda mayores posibilidades de supervivencia en el mercado, además de los impactos que pueden generar en el entorno en el que se desarrollan.

\subsection{La integración social y las redes de cooperación inter-empresarial en entornos turísticos rurales}

El turismo en el ámbito rural juega un papel estratégico como elemento dinamizador del desarrollo económico y social debido a que está directamente vinculado con la valorización, integración y aprovechamiento de los recursos territoriales (Tolstad, 2014; Quaranta et al., 2016). Las Mypes y los emprendimientos comunitarios son parte importante del proceso de desarrollo rural; no obstante, se trata de organizaciones que -en su mayoríamantienen bajos niveles de innovación y que dependen de otros sectores para ser innovadoras, además de las diversas desventajas que enfrentan con relación a las condiciones del entorno donde se desempeñan (Hjalager, 2002).

Las $\mathrm{RCl}$ constituyen una vía que facilita el uso eficientemente de los recursos locales, la movilización de las ideas, la activación de las capacidades empresariales y el emprendimiento de dinámicas económicas y sociales capaces de crear relaciones simbióticas entre las organizaciones y el entorno local (Tolstad, 2014; Seelos et al., 2015). Para algunos autores, las $\mathrm{RCl}$ encuentran su potencial de innovación en las relaciones entre emprendedores $y$, particularmente, en factores como la confianza mutua, la proximidad física, social y 
profesional e incluso en los valores y preocupaciones colectivas (Becattinni, 1988) que son provistas, casi de manera natural, por el contexto local.

El enfoque de la integración social (social embeddedness en inglés) propuesto por Granovetter (1985) y Polanyi (1992), permite explicar cómo las organizaciones operan en términos de normas compartidas, cooperación, interacción y confianza para transformar los recursos generados por las propias redes para acceder de forma individual y colectiva a las oportunidades disponibles en el entorno (Cooke, 2001; Booyens y Rogerson, 2016). Este abordaje muestra cómo la combinación de los recursos sociales y las habilidades empresariales podrían dar lugar a formas de innovación que van más allá de la racionalidad económica, debido a su capacidad para generar cambios sociales.

La integración social (IS) es un enfoque necesario en el estudio del turismo rural ya que permite analizar las redes inter-empresariales no sólo como estrategias comerciales sino como sistemas de cooperación ampliamente influenciadas por las relaciones sociales diádicas y de red. Algunos estudios plantean que las Mypes y los emprendimientos comunitarios podrían operar bajo una intensa dinámica de red, siempre y cuando sus actividades económicas estén impulsadas por sistemas de relaciones sociales que son base para la generación de ideas, el intercambio de conocimientos y la innovación (Greenberg et al., 2018; Wigren et al., 2019; Tregear y Cooper, 2016).

Si bien son numerosas las investigaciones que reconocen la existencia de ciertos factores determinantes para la IS de los emprendedores en $\mathrm{RCl}$, pocas son las que tratan de identificarlos en el ámbito del turismo y, más aún, en entornos rurales. Siendo así, las escasas publicaciones identifican la confianza como un generador de expectativas positivas que facilitan las relaciones y la cooperación inter-empresarial (Czernek y Czakon, 2016; Granovetter, 1985; Hite, 2005). Otros trabajos enfatizan en el capital social, argumentando que las acciones y la decisión de cooperación están impulsadas por las normas sociales y los valores (Díez y Monto, 2014; Mu et al., 2008). Incluso algunos trabajos apuntan a la proximidad espacial, particularmente, porque induce a la creación de relaciones densas y a la transferencia de conocimiento tácito (Booyens y Rogerson, 2016; Sorensen, 2007).

Con relación a las ventajas de integración, se demuestra que la incorporación en redes locales puede mejorar la habilidad del emprendedor para acceder a ciertos recursos como la información, el conocimiento local, la legitimación social y, particularmente, la innovación (Burt, 2001; Brenner et al., 2013). Algunos otros muestran que las organizaciones fuertemente arraigadas al contexto social tienen mayores probabilidades de superar las limitaciones institucionales y de mercado (Díez y Monto, 2014; Tregear y Cooper, 2016). Si bien son diversas las ventajas que provee la IS de los emprendedores en $\mathrm{RCl}$, las más reconocidas son: el acceso a recursos materiales y económicos, el respaldo simbólico del entorno social, nueva información y conocimientos, entre otros (Faccin et al., 2017; Czernek, 2020).

Siendo la IS un proceso que permite a las organizaciones turísticas acceder a las oportunidades que les brinda el entorno rural, surgen las siguientes preguntas: ¿cuáles son los factores que, en las relaciones sociales, facilitan el proceso de integración de las empresas turísticas en redes de cooperación inter-empresarial en entornos rurales?, ¿cuáles son las ventajas que esos factores de integración aportan a los emprendedores turísticos en su participación en redes de cooperación? 


\section{METODOLOGÍA}

En esta investigación se realizó un análisis relacional que ofrece una visión general de los artículos académicos centrados en el tema de estudio, principalmente, de revistas internacionales en idioma inglés, publicados del año 2000 hasta la actualidad y que se encontraron en plataformas como: Google Académico, Elservier, Taylor y Francis Online y Emerald Insight. Los primeros estudios sobre IS surgen a mediados de la década de los noventa, pero no se trató de estudios empíricos enfocados en la actividad turística ni en entornos rurales. Se identificó que los primeros trabajos de IS en el ámbito del turismo rural surgen a principios del año 2000. La primera búsqueda se realizó en Google Académico utilizando la consulta social embeddedness and rural tourism. Fueron analizados los resúmenes de los primeros 44 resultados y sólo 20 artículos cumplieron los criterios mencionados. Debido a la escasez de estudios que vinculen la IS, el turismo y el ámbito rural, se realizó una segunda búsqueda utilizando el resto de las plataformas mencionadas. La consulta se enfocó en artículos que tuvieran las siguientes asociaciones: social embeddedness and rural tourism, relational embeddedness and rural tourism entrepreneurship, inter-firm networks and rural tourism, networking embeddedness and SMEs and rural context, overembeddedness, entre otros. Esto condujo a la obtención de 123 artículos, siendo seleccionados 72 documentos, analizando un total de 92 artículos de revistas científicas internacionales.

Para el análisis, se optó por un diseño metodológico apegado al Análisis Temático (AT) (Braun y Clarke, 2006). Este método permitió identificar, analizar e informar patrones (temas) dentro de los datos recolectados, los cuales fueron identificados a través de un proceso deductivo; es decir, su selección fue impulsada por un interés teórico planteado previamente. Se crearon dos categorías principales: por un lado, los factores de las relaciones sociales que facilitan la integración de los emprendedores en redes inter-empresariales y, por el otro, las ventajas que estos factores aportan a los emprendedores en sus relaciones de cooperación. Para la codificación se utilizó el software Atlas. ti. Después de un proceso de comparación, fusión y rechazo de códigos, se crearon 16 subcódigos para la primera categoría y 25 para la segunda (Ver Tabla 1) 
Tabla1. Códigos que responde a los factores y ventajas de IS

\begin{tabular}{|c|c|c|}
\hline FACTORES DETERMINANTES & \multicolumn{2}{|c|}{ VENTAJAS DE INTEGRACIÓN SOCIAL } \\
\hline A1. Fuertes lazos locales & $\begin{array}{l}\text { B1. Alto potencial para vincular producción } \\
\text { y consumo }\end{array}$ & $\begin{array}{l}\text { B18. Facilita el acceso e intercambio de productos } \\
\text { y servicios entre firmas }\end{array}$ \\
\hline A2. Lazos externos & B2. Actores dispuestos a tomar riesgos & $\begin{array}{l}\text { B19. Puede desarrollar mercados laborales y } \\
\text { servicios de insumos especializados }\end{array}$ \\
\hline A3. Sistema económico abierto & B3. Acceso a nuevos recursos & B20. Puede desarrollar capacidades colectivas \\
\hline A4. Sistema abierto al conocimiento & B4. Identidad y objetivos comunes & $\begin{array}{l}\text { B21. Puede generar reglas informales que facilitan } \\
\text { la coordinación entre empresas }\end{array}$ \\
\hline A5. Relaciones de confianza & $\begin{array}{l}\text { B5. Reducción en el costo de las } \\
\text { transacciones }\end{array}$ & B22. Elimina la posibilidad conductas oportunistas \\
\hline A6. Localismo & $\begin{array}{l}\text { B6. Es soporte para la adaptación y difusión } \\
\text { de innovaciones }\end{array}$ & B23. Impulsa la conducta emprendedora \\
\hline A7. Normas y valores compartidos & $\begin{array}{l}\text { B8. Reduce los costos de establecer } \\
\text { relaciones de cooperación }\end{array}$ & B24. Genera economías resilientes \\
\hline A8. Prácticas de asociación y cooperación & $\begin{array}{l}\text { B9. Reduce los costos en las transacciones } \\
\text { de mercado }\end{array}$ & $\begin{array}{l}\text { B25. Facilita el intercambio de conocimiento e } \\
\text { información }\end{array}$ \\
\hline A9. Integración geográfica & $\begin{array}{l}\text { B10. Reduce los costos por control y } \\
\text { monitoreo de los socios }\end{array}$ & \\
\hline A10. Comunicación & B11. Desarrolla la empatía & \\
\hline $\begin{array}{l}\text { A11. Relaciones formales con otros } \\
\text { actores sociales }\end{array}$ & $\begin{array}{l}\text { B12.Facilita el inicio y aceptación de } \\
\text { emprendedores en el entorno }\end{array}$ & \\
\hline A12. Descentralización del gobierno local & B13. Facilita la coordinación entre firmas & \\
\hline A13. Regulaciones gubernamentales & B14.Permite alcanzar ventajas competitivas & \\
\hline A14. Liderazgos locales & $\begin{array}{l}\text { B15. Permite la solución flexible a } \\
\text { problemas de cooperación }\end{array}$ & \\
\hline A15. Relaciones informales & B16. Crea una cultura de cooperación & \\
\hline $\begin{array}{l}\text { A16. Complementan la oferta turística de } \\
\text { otros }\end{array}$ & B17. Reduce costos/cercanía geográfica & \\
\hline
\end{tabular}

Elaboración propia

Conocer la interrelación conceptual de los códigos forma parte del objetivo de esta investigación. El software Atlas.ti ofrece una herramienta para reconocer los patrones de interconexión entre los códigos denominada tabla de co-ocurrencias; si bien esta herramienta hace posible reconocer las interconexiones entre los códigos, no permite observar las "comunidades" subyacentes en las que se encuentran los códigos interconectados. Aprovechando los avances en el campo del análisis de redes, se utilizó la tabla de coocurrencias generada por Atlas. ti como una matriz de adyacencia misma que es la base para visualizar gráficamente la red de códigos, aunque no las comunidades subyacentes. Para ello, se aplicó la métrica de modularidad a la red con el software Gephi; revelándose así tres comunidades de códigos subyacentes, siendo dos ( $\mathrm{A}$ y $\mathrm{B}$ ) las más relevantes por el número de nodos y relaciones que reúnen. Además de aplicar la métrica de modularidad a la red, también se utilizó el algoritmo de esquema ForceAtlas2 para visualizar gráficamente las comunidades de códigos. 


\section{RESULTADOS}

\subsection{Factores de integración social (IS) a redes de cooperación inter-empresarial}

El proceso de IS tiene un rol importante en la creación de ambientes de cooperación entre los emprendedores turísticos, lo cual también puede contribuir en la generación de productos, servicios y destinos innovadores. En la revisión de la literatura, se identificaron seis factores que con mayor frecuencia aparecen como determinantes de la IS de los emprendedores a $\mathrm{RCl}$, estos son: la existencia de fuertes lazos locales (A1), los lazos o relaciones con actores externos $(A 2)$, las relaciones de confianza $(A 5)$, las normas y valores compartidos (A7), las prácticas de asociación y cooperación, y las relaciones informales (A15); siendo los dos primeros $(A 1, A 2)$ los más referidos.

Al respecto, algunos autores afirman que la búsqueda de la proximidad social entre emprendedores y entre estos y otros actores sociales es un factor capaz de promover la cooperación y la innovación (Bachinger, 2011; Brenner et al., 2013). Para el caso de los emprendedores turísticos en el ámbito rural, se identificó que las relaciones personales e informales con un alto contenido de confianza, reciprocidad, solidaridad, normas y creencias compartidas, actúan como mecanismos de integración económica y social (Kofler et al., 2018; Kofler y Marcher, 2018; Saxena, 2005). En el ámbito del turismo, estas redes personales pueden estar conformadas por las personas con las cuales un emprendedor tiene relaciones directas, ejemplo: proveedores, distribuidores, socios, empresarios, algunas asociaciones comerciales o civiles e incluso clientes.

Aunque, para algunos autores este tipo de vínculos solo resultan útiles en las primeras etapas del emprendimiento (Cátia y Franco, 2016; Dredge, 2006); para otros, dichas relaciones desempeñan un papel crucial durante el ciclo de la vida empresarial (Novelli et al., 2006; van der Zee y Vanneste, 2015). Se encontró un fuerte patrón de asociación entre los fuertes lazos locales (A1), las relaciones informales (A5) y las relaciones de confianza (A15) (Ver Anexo A). La interrelación entre estos factores puede ser explicada de diversas formas; por ejemplo, algunos autores (Czernek, 2014, 2020; Kelliher et al., 2018; Shockley et al., 2000) mencionan que las relaciones cercanas entre empresas y su integración en $\mathrm{RCl}$ incrementa cuando los emprendedores se perciben como honestos y confiables. Otros mencionan que los intensos lazos locales producen cierto grado de "confianza exigible", que refiere a la capacidad de dichos lazos para obligar a los emprendedores a cumplir con las normas y valores aceptados por el colectivo (Seelos et al., 2015; Tolstad, 2014). Incluso hay quienes afirman que altos niveles de confianza entre emprendedores facilita encontrar intereses en común que, generalmente, desembocan en redes de cooperación (Kofler et al., 2018; Saxena, 2005).

De acuerdo con los resultados, la IS de los emprendedores en RCl también está determinada por las normas y valores compartidos (A7). La mayoría de las investigaciones (Scott, 2001; Anderson y Miller, 2003), conciben estos factores como modelos compartidos de naturaleza formal e informal que determinan el estándar de comportamiento apropiado y esperado por los integrantes de un grupo dentro de una actividad económica; de tal forma que estos factores (A7) actúan como formas de control social evitando, por ejemplo, prácticas abusivas entre emprendedores.

En el análisis de la literatura, se observa otro patrón de asociación entre las normas y valores compartidos (A7), las prácticas de asociación y cooperación (A8) y las relaciones de confianza (A5) (Ver Tabla 2). De acuerdo con la revisión, las normas y valores crean un orden 
social en la lógica de trabajo, la toma de decisiones y, en general, regulan las alianzas, el intercambio de recursos y las relaciones de cooperación entre emprendedores turísticos (Bramwell y Lane, 2000; Lemmetyinen y Frank, 2009; Wang y Fesenmaier, 2007). Se encuentra coincidencia en que las normas y valores (A7) son factores que promueven formas de entendimiento común (Anderson y Jack, 2002; Jenssen y Koenig, 2002), mismas que son necesarias para la generación y mantenimiento de las redes de cooperación a largo plazo (Ramayah et al., 2011). Particularmente, las relaciones de confianza y la informalidad implícita juegan un papel clave en la disposición de los actores para la cooperación debido a que reducen el riesgo económico causado por conductas oportunistas (Díez y Montoro, 2014; Kofler et al., 2018; Mu et al., 2008).

Tabla 2. Co-ocurrencias entre los factores determinantes de la integración social

\begin{tabular}{|c|c|c|c|c|c|c|c|c|c|c|c|c|c|c|c|c|c|}
\hline & & A1 & A2 & A3 & A4 & A5 & A6 & A7 & A8 & A9 & A10 & A11 & A12 & A13 & A14 & A15 & A16 \\
\hline $\mathrm{A} 1$ & Fuertes lazoslocales & 0.00 & 0.41 & 0.00 & 0.00 & 0.06 & 0.01 & 0.03 & 0.02 & 0.03 & 0.00 & 0.01 & 0.00 & 0.00 & 0.00 & 0.12 & 0.00 \\
\hline A2 & $\begin{array}{l}\text { Lazos } \\
\text { externos/empresasagentes }\end{array}$ & 0.41 & 0.00 & 0.00 & 0.00 & 0.01 & 0.00 & 0.01 & 0.02 & 0.01 & 0.00 & 0.03 & 0.00 & 0.01 & 0.01 & 0.02 & 0.00 \\
\hline A3 & Sistema económico abier to & 0.00 & 0.00 & 0.00 & 0.00 & 0.00 & 0.00 & 0.00 & 0.00 & 0.00 & 0.00 & 0.00 & 0.00 & 0.00 & 0.00 & 0.00 & 0.00 \\
\hline A4 & Sistema abierto al conocimiento & 0.00 & 0.00 & 0.00 & 0.00 & 0.00 & 0.00 & 0.00 & 0.00 & 0.01 & 0.00 & 0.00 & 0.00 & 0.00 & 0.00 & 0.01 & 0.00 \\
\hline A5 & Relaciones de confianza & 0.06 & 0.01 & 0.00 & 0.00 & 0.00 & 0.00 & 0.14 & 0.10 & 0.02 & 0.02 & 0.00 & 0.00 & 0.00 & 0.00 & 0.03 & 0.00 \\
\hline $\mathrm{A} 6$ & Rurality/locality & 0.01 & 0.00 & 0.00 & 0.00 & 0.00 & 0.00 & 0.01 & 0.00 & 0.01 & 0.00 & 0.00 & 0.00 & 0.00 & 0.00 & 0.00 & 0.00 \\
\hline A7 & Normas, valorescompartidos & 0.03 & 0.01 & 0.00 & 0.00 & 0.14 & 0.01 & 0.00 & 0.19 & 0.00 & 0.02 & 0.00 & 0.00 & 0.00 & 0.01 & 0.00 & 0.00 \\
\hline A8. & $\begin{array}{l}\text { Prácticas de asociación y } \\
\text { cooperación }\end{array}$ & 0.02 & 0.02 & 0.00 & 0.00 & 0.10 & 0.00 & 0.19 & 0.00 & 0.00 & 0.02 & 0.00 & 0.00 & 0.00 & 0.00 & 0.01 & 0.00 \\
\hline A9. & Integración geográfica & 0.03 & 0.01 & 0.00 & 0.01 & 0.02 & 0.01 & 0.00 & 0.00 & 0.00 & 0.01 & 0.01 & 0.00 & 0.00 & 0.00 & 0.00 & 0.00 \\
\hline A10 & Comunicación & 0.00 & 0.00 & 0.00 & 0.00 & 0.02 & 0.00 & 0.02 & 0.02 & 0.01 & 0.00 & 0.00 & 0.00 & 0.00 & 0.00 & 0.01 & 0.00 \\
\hline A11 & $\begin{array}{l}\text { Relacionesformales con otros } \\
\text { actoressociales }\end{array}$ & 0.01 & 0.03 & 0.00 & 0.00 & 0.00 & 0.00 & 0.00 & 0.00 & 0.01 & 0.00 & 0.00 & 0.00 & 0.00 & 0.01 & 0.18 & 0.00 \\
\hline A12 & $\begin{array}{l}\text { Descentralización del gobierno } \\
\text { local }\end{array}$ & 0.00 & 0.00 & 0.00 & 0.00 & 0.00 & 0.00 & 0.00 & 0.00 & 0.00 & 0.00 & 0.00 & 0.00 & 0.00 & 0.00 & 0.00 & 0.00 \\
\hline A13 & Regulaciones gubernamentales & 0.00 & 0.01 & 0.00 & 0.00 & 0.00 & 0.00 & 0.00 & 0.00 & 0.00 & 0.00 & 0.00 & 0.00 & 0.00 & 0.00 & 0.00 & 0.00 \\
\hline A14 & Lider azgos locales & 0.00 & 0.01 & 0.00 & 0.00 & 0.00 & 0.00 & 0.01 & 0.00 & 0.00 & 0.00 & 0.01 & 0.00 & 0.00 & 0.00 & 0.00 & 0.00 \\
\hline A15 & Relacionesinformales & 0.12 & 0.02 & 0.00 & 0.01 & 0.03 & 0.00 & 0.00 & 0.01 & 0.00 & 0.01 & 0.18 & 0.00 & 0.00 & 0.00 & 0.00 & 0.00 \\
\hline A16 & $\begin{array}{l}\text { Complementan la oferta turística } \\
\text { de otros cooperación }\end{array}$ & 0.00 & 0.00 & 0.00 & 0.00 & 0.00 & 0.00 & 0.00 & 0.00 & 0.00 & 0.00 & 0.00 & 0.00 & 0.00 & 0.00 & 0.00 & 0.00 \\
\hline
\end{tabular}

Elaboración propia

Finalmente están los lazos externos (A2). Aunque algunos autores (Granovetter, 1985; Burt, 2001), los consideran como una forma de afianzar la integración social de los emprendedores a las RCl locales debido a que pueden incrementar la sensación de lo colectivo (Burt, 2001); en el contexto del turismo rural son aún más reconocidos por su capacidad para vincular a los emprendedores con grupos externos y, de esta manera, acceder a nuevas ideas e información que pueden desembocar en innovaciones (Jack y Anderson, 2002; Tolstad, 2014; Booyens y Rogerson, 2016). 


\subsection{Las ventajas de integración social (IS) para la cooperación inter-empresarial}

Este segundo análisis se centró en identificar las ventajas que los factores de integración aportan a los emprendedores para su participación en redes de cooperación. Entre las relaciones más sobresalientes, se encontró que la existencia de fuertes lazos locales (A1) y de lazos externos (A1) establecen las condiciones para que los emprendedores turísticos accedan a nuevos recursos (B3), además de que son soporte para la adaptación y difusión de innovaciones (B6), facilitan la coordinación entre empresas (B13), crean una cultura de cooperación (B16) y propician el intercambio de conocimientos e información (B25) (Ver Anexo A).

A través del análisis de la modularidad, se identificó que los factores y las ventajas de integración conforman dos comunidades de códigos relevantes (Ver Figura 1). En la primera (Comunidad A), los fuertes lazos locales (A1) y los lazos externos (A2) se colocan como los principales factores de IS y entre las ventajas más importantes están el acceso a nuevos recursos (B3), la adaptación y difusión de innovaciones (B6) y el intercambio de conocimientos e información (B25). Con relación a los documentos revisados, se interpreta que los lazos fuertes locales (A1) son una forma de integración de los emprendedores al sistema productivo local (Beritelli, 2011; Booyens y Rogerson, 2017) y, los lazos externos más que un mecanismo para afianzarse a las redes locales, son un medio para integrarse a redes de cooperación a escala regional, nacional e incluso internacional con fines de innovación (Díez y Monto, 2014; Narváez y Fernández, 2013).

Figura 1. La red completa y la Comunidad A de códigos

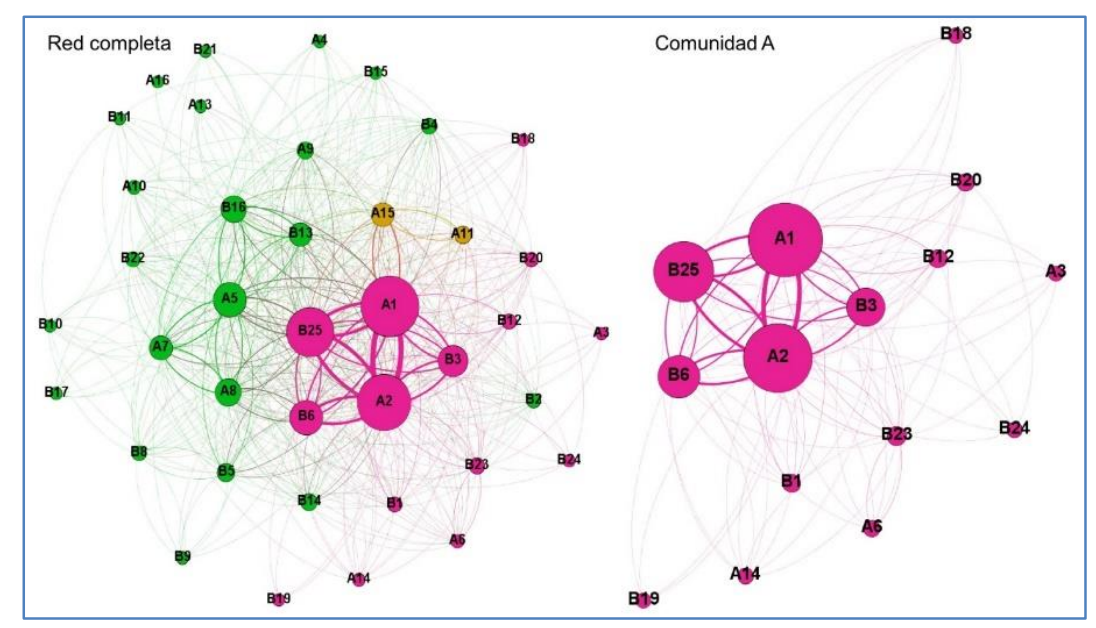

Elaboración propia

A pesar de que ambos factores (A1) y (A5) se muestran como centrales en esta comunidad (A), las correlaciones más intensas se muestran entre los lazos fuertes locales (A1) y la mayoría de las ventajas enunciadas anteriormente (Ver Anexo A). Estos factores son altamente valorados porque generan diversas ventajas para los emprendedores, tales como la transferencia de conocimientos, generalmente, de naturaleza tácita (Farrell et al., 2010; Zhang, 2010), son soporte emocional para los nuevos emprendedores (Skokic et al., 2019; Kimbu y Ngoasong, 2016), facilitan la interacción y cooperación (Bachinger, 2011; Divisekera y Nguyen, 2011; Kofler y Marcher, 2018) e incluso algunos trabajos los colocan como determinantes en la innovación turística (Brenner et al., 2013; van der Zee y Vanneste, 2015). 
Aunque con grados de interrelación más bajos, en esta comunidad también se enfatiza en los lazos con agentes externos (A2). Algunos de los planteamientos, suponen que la IS de los emprendedores en redes con una alta diversidad de actores (Brandão, et al., 2019) posibilita el acceso a recursos, mercados y sistemas de tecnología más amplios que los existentes en las redes locales (Powell y Grodal, 2006; Kofler y Marcher, 2018). Este tipo de redes facilitan el intercambio de conocimientos y la difusión de información entre empresas y entre estas y otros actores (Booyens y Rogerson, 2017; Martínez et al., 2019). Incluso incentivan al emprendedor para mantener mayor interacción con el mercado (Inkpen y Tsang, 2005; Greenberg et al., 2018) o la formación de alianzas formales para acceder -entre otros recursos - a clientes potenciales (de Klerk y Saayman, 2012).

La segunda comunidad de códigos (Comunidad B), agrupa aquellos que aluden a la integración de los emprendedores turísticos en relaciones diádicas entre emprendedores; por tanto, las ventajas de la integración de los emprendedores son útiles para su desempeño en el ámbito local. Se encontró que las relaciones de confianza (A5), las prácticas de asociación y cooperación (A8), las normas y valores compartidos (A7) y la integración geográfica (A9) aparecen en esta comunidad de códigos como los factores de IS más relevantes. Igualmente, entre las ventajas más notables que producen dichos factores están la creación de una cultura de cooperación (B16), la coordinación entre empresas (B13), la reducción en el costo de las transacciones (B5) y el logro de las ventajas competitivas (B14), entre otros efectos que aparecen con menor frecuencia (Ver Figura 2).

Figura 2. Comunidad B de códigos

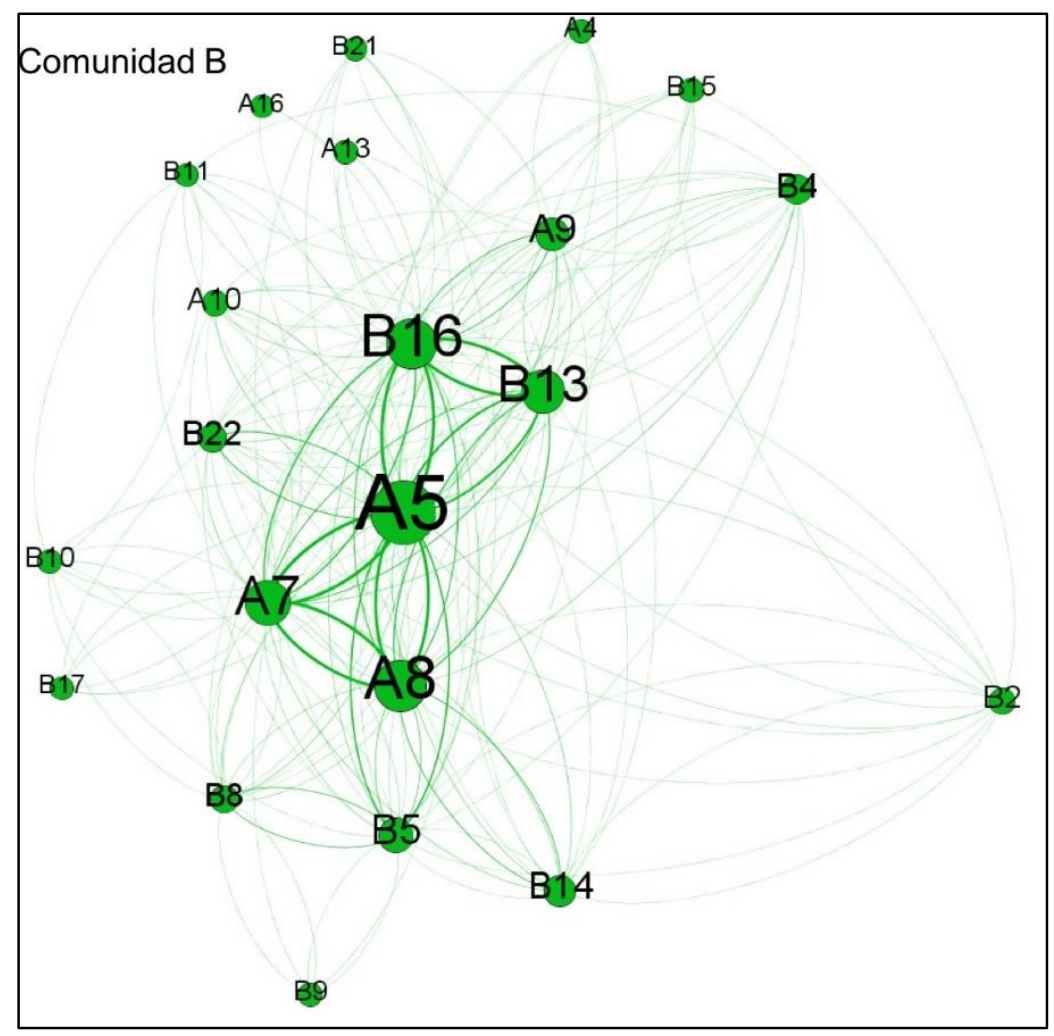

Elaboración propia 
Se observa que tanto las relaciones de confianza (A5) como las prácticas de asociación y cooperación (A8) y las normas y valores compartidos (A7) son factores que están frecuentemente asociados a formas de capital social, las cuales son esenciales en las dinámicas inter-empresariales en el ámbito local (Huggins, 2000; Laschewski et al., 2002). Específicamente, se argumenta que los destinos turísticos en los que el sistema productivo local experimenta una alta densidad de relaciones mediadas por dichos factores, son buenas "plataformas" para el desarrollo de redes de cooperación (Czernek, 2014; Proctor et al., 2012), la principal razón es que los destinos en el ámbito rural aún se caracterizan por poblaciones estables y de larga permanencia, se cree que estos espacios aún brindan oportunidades de intimidad y confianza para el desarrollo de relaciones sociales intensas.

Los resultados demuestran que estos factores $(A 5, A 7, A 8)$ tienen una fuerte influencia en la creación de una cultura de cooperación (B16) y en la coordinación entre los emprendedores (B13). Para algunos autores, los altos niveles de confianza son la base del entendimiento mutuo y reciprocidad entre emprendedores turísticos (Stoddarta et al., 2019; Weidenfeld, 2013). En esta correspondencia, las normas y valores (A7) proveen una sistemas de comprensión y significados que guian el comportamiento, este caso, sobre los permisos o límites en las relaciones de cooperación; incluso hay quienes afirman que tanto la confianza así como la práctica de normas y valores contribuyen a eliminar las conductas oportunistas de alguno de los integrantes de la red (Czernek, 2020; Kelliher et al., 2018). Finalmente, las prácticas de asociación y cooperación (A8) hacen de la cultura de cooperación un proceso de transacciones fundado en las relaciones cercanas, repetidas y de negociación voluntaria (Cátia y Franco, 2016; Kofler et al., 2018; Saxena, 2005).

En esta comunidad (B), la integración geográfica (A9) mantiene una fuerte relación con la cultura de cooperación (B16); además de referir a la cercanía física, la proximidad geográfica alude implícitamente a la proximidad relacional y cultural de los emprendedores (Czernek, 2017; Novelli et al., 2006), que facilita la construcción de redes de cooperación. Para algunos autores, este factor (A9) estimula las iniciativas conjuntas del día a día entre emprendedores (Hjalager, 2002; Kofler el al., 2018; Weidenfeld, 2013), aunque también destaca la cooperación entre el emprendedor y los miembros de su familia ya que los negocios turísticos en el entorno rural, generalmente, se caracterizan por su cercanía con la casa del emprendedor (Tolstad, 2014; Tregear y Cooper, 2016). Si bien, son claras las implicaciones de la proximidad geográfica, es importante destacar que también guarda una relación intensa con ventajas que no estan consideradas en esta comunidad, por ejemplo, el intercambio de conocimientos e información entre emprendedores (B25).

Desde la perspectiva de la IS, tambien se observa la influencia de estos factores (A5, $A 7, A 8)$ sobre la reducción en el costo de las transacciones (B5). Por un lado, las relaciones cercanas (las de confianza) influyen en los emprendedores turísticos para conformar $\mathrm{RCl}$ que se basan en el "trueque" como mecanismo de intercambio de recursos (Czernek y Czakon, 2016; Czernek, 2020); es decir, los lazos sociales impulsan las colaboraciones económicas voluntarias al tiempo que reducen riesgos (Uzzi, 2000; Ying-Hueih et al., 2014). Por otro lado, las relaciones cercanas reducen los costos de transacción al facilitar la cooperación, evitando el gasto de recursos (tiempo, dinero, negociaciones) en la búsqueda y elección de un socio o emprendedor "con quien cooperar"(Bachinger, 2011; Beaumont y Dredge, 2010).

La revisión de los documentos, muestra que las relaciones cercanas $(A 5, A 7, A 8)$ tienen un papel fundamental en la generación de ventajas competitivas (B14). Algunos autores, 
observan la influencia que ejercen para convertir la proximidad social en "fuerzas productivas" (Faccin et al., 2017, p.57), pues impulsan el desarrollo de estrategias comunes (Anderson y Jack, 2002; Cátia y Franco, 2016), fortalezcen la difusión de conocimiento y el intercambio de experiencias y recursos locales para la creación de productos y servicios turísticos innovadores (Beaumont y Dredge, 2010; Kofler et al., 2018; Soisalo y Lindroth, 2006), entre otras ventajas.

\section{DISCUSIÓN Y CONCLUSIONES}

La idea básica de la perspectiva de la IS y de las $\mathrm{RCl}$ es la de considerar a los emprendedores turísticos integrados a una estructura relacional para diseñar mecanismos colectivos que, en conjunto con otros emprendedores, les permitan adquirir, crear y compartir información y otros recursos necesarios para competir en el mercado a través de productos, servicios y destinos innovadores. Este trabajo indaga en los factores que incentivan a los emprendedores turísticos en el ámbito rural para integrarse a redes de cooperación interempresarial y, al mismo tiempo, se observan las ventajas que estos factores producen sobre sus relaciones de cooperación.

Respecto a lo primero, en la revisión y el análisis de la literatura realizada se destacan aquellos factores que, predominantemente, son resultado de la proximidad geográfica, social y cultural característica de los entornos rurales. En este trabajo de investigación se observa coincidencia entre los supuestos teóricos y el estudio empírico de la IS. Los trabajos revisados colocan los lazos locales, la confianza, las prácticas de asociación y las relaciones informales como los principales factores que influyen en la IS de los emprendedores a $\mathrm{RCl}$; por tanto, puede afirmarse que es la similitud o proximidad entre actores y organizaciones el hecho o circunstancia que dinamiza las relaciones cercanas y repetidas entre los emprendedores turísticos (Booyens y Rogerson, 2016; Sorensen, 2007) y, por supuesto, es un hecho determinante en su integración a $\mathrm{RCl}$. Al igual que la confianza, los valores y normas compartidas emergen en el análisis como factores determinantes para la integración de los emprendedores en $\mathrm{RCl}$; particularmente, se situan como esquemas del comportamiento apropiado que deben mostrar los emprendedores para crear escenarios de colaboración.

Aunque, gran parte de las investigaciones revisadas argumentan a favor de los lazos locales fuertes como impulsores de la innovación turística (Albrecht, 2013; Brandão et al., 2019; Czernek, 2020; Hall, 2005); el análisis de la bibliografía evidencia que son las redes de cooperación con agentes externos las que propician los procesos de innovación; en este caso, porque los emprendedores encuentran un mayor acceso a recursos "novedosos" y actualizados que no estan basados en relaciones fuertes y duraderas. Incluso, también queda en evidencia que el intercambio de conocimiento y la difusión de innovaciones está dada por la posibilidad de los emprendedores para establecer vínculos de cooperación con otros emprendedores fuera del entorno local (ver Anexo A).

El análisis de la modularidad permitió analizar las ventajas que encuentran los emprendedores en su integración a $\mathrm{RCl}$. Siendo así, se revelan ventajas para la cooperación inter-empresarial a nivel del entorno local y, también para la vinculación con emprendedores a otras escalas. Se observa que las primeras; es decir, la coordinación entre empresas, la cultura de cooperación y el intercambio de conocimientos crean condiciones a nivel de las relaciones cercanas facilitando la sinergia entre emprendedores y la cotinuidad de la cooperación inter-empresarial. Las segundas (acceso a nuevos recursos y soporte para la adaptación y difusión de innovaciones), son ventajas que se surgen de la cooperación con 
actores a escala regional, nacional e incluso internacional y que, de acuerdo con la literatura revisada, propician la innovación (Ver Figura 1 y 2).

El estudio de las redes también permite reconocer que las $\mathrm{RCl}$ en el ámbito rural pueden ser propensas a un efecto de sobreintegración social (overembeddeness) que refiere a que las relaciones intensas, de larga duración y mediadas por factores como la confianza o la reciprocidad en extremo pueden conducir a que los emprendedores ignoren la información, el conocimiento y las formas de innovación provenientes de las relaciones extralocales. Esta idea abre la posibilidad de indagar en las desventajas que las $\mathrm{RCl}$ a nivel local generan entre los emprendedores, esta premisa se situa como una de las posibles líneas de investigación que propone este trabajo de investigación. Aunque, produndizar sobre los factores determinantes y las ventajas que generan las redes extralocales también se visualiza como una prioridad en el campo de las $\mathrm{RCl}$ en el ámbito rural.

Finalmente, se identifica que aunque el trabajo trata de reunir un número significativo de trabajos sobre la temática y extraerlos de las bases más utilizadas por la comunidad científica a nivel mundial; se reconoce que mantiene un nivel exploratorio.

\section{REFERENCIAS BIBLIOGRÁFICAS}

Albrecht, J. (2013). Networking for sustainable tourism-towards a research agenda, Journal of $\begin{array}{llll}\text { Sustainable } \quad \text { Tourism, } & \text { 639-657. }\end{array}$ https://doi.org/10.1080/09669582.2012.721788

Anderson, A., y Miller, C. (2003). "Class matters": human and social capital in the entrepreneurial process, Journal of Behavioral and Experimental Economics, 32 (1), 17-36. doi: 10.1016/S1053-5357(03)00009-X

Anderson, A., y Jack, S. (2002). The articulation of social capital in entrepeneurial networks: a glue or a lubricant?, Entrepreneurship y Regional Development: An International Journal, 14 (3), 193-210. doi: 10.1080/08985620110112079

Bachinger, M. (2011). Regions and networks: models of cooperation on the competitiveness of a region. Wiesbaden: Springer.

Beaumont, N. y Dredge, D. (2010). Local tourism governance: a comparison of three network approaches. Journal of Sustainable Tourism, 18 (1), 7-28. doi: 10.1080/09669580903215139

Becattinni, G. (1988). Los Distritos Industriales y el reciente Desarrollo Italiano . Sociología del Trabajo, 5, 3-17.

Beritelli, P. (2011). Cooperation among prominent actors in a tourist destination. Annals of Tourism Research, 38 (2), 607-629. doi: 10.1016/j.annals.2010.11.015

Booyens, I., y Rogerson, C. (2016). Networking and learning for tourism innovation: evidence from the Western Cape. Tourism Geographies, 19 (3), 1-21.

Booyens, I., y Rogerson, C. (2017). Networking and learning for tourism innovation evidence from the Western Cape. Tourism Geographies. An International Journal of Tourism Space, Place and Environment, 19 (3), 340-361.doi: 10.1080/14616688.2016.1183142

Bramwell, B., y Lane, B. (2000). Tourism Collaboration and Patherships. Politics, Practice and Sustainability. Gran Bretaña: Channel View Publications. 
Brandão, F., Breda, Z. B. y Costa, C. (2019). Innovation and internationalization as development strategies for coastal tourism destinations: The role of organizational networks. Journal of Hospitality and Tourism Management, 41, 219-230. doi: 10.1016/j.jhtm.2019.10.004

Brandão, F., Breda, Z., y Costa, C. (2019). Innovation and internatinalization as developmento strategies for coastal tourism destinations: The role of organizational networks. Journal of Hospitality and Tourism Management, 41, 1-12. doi: 10.1016/j.jhtm.2019.10.004

Braun, V., y Clarke, V. (2006). Using thematic analysis in Psychology. Qualitative Research in Psychology, 3 (2), 77-101. doi:10.1191/1478088706qp063oa

Brenner, T., Cantner, U., y Graf, H. (2013). Introduction: Structure and Dynamics of Innovation Networks. Regional Studies, 47(5), 647-650. doi: 10.1080/00343404.2013.770302

Burt, R. (2001). The Social Capital of Structural Holes. En M. Guillien, R. Collins, P. England, y M. Meyer, New directions in economic sociology. New York: Russell Sage Foundation.

Cátia, J., y Franco, M. (2016). Cooperation networks in toruism: A study of hotels and rural tourism establishments in an inland region of Portugal. Journal of Hispitality and Tourism Mangement, 29, 165-175. doi: 10.1016/j.jhtm.2016.07.005

Cooke, P. (2001). Regional innovations systems, clusters and knowwledge economy. Industrial and Corporate Change, 10(4), 945-973. doi: 10.1093/icc/10.4.945

Czernek, K. (2014). The role of social embeddedness in touristregion cooperation. European Journal of Tourism, Hospitality and Recreation, 5 (2), 61-81.

Czernek, K. (2017). Tourism features as determinants of knowledge tranfer in the process of tourist cooperation. Current Issues in Tourism, 20, 204-220. doi: 10.1080/13683500.2014.944107

Czernek, K. (2020). Social embeddedness and its benefits for cooperation in a tourism destination. Journal of Destination Marketing y Management, 15, In press. doi: 10.1016/j.jdmm.2019.100401

Czernek, K., y Czakon, W. (2016). Trust-building processes in tourist coopetition: The case of a Polish region. Tourism Management, 52, 380-394 doi: 10.1016/j.tourman.2015.07.009

de Klerk, S., y Saayman, M. (2012). Networking as a key factor in Artpreneurial success. European Business Review , 24 (5), 382-399. doi: 10.1108/09555341211254490

Díez, I., y Monto, Á. (2014). Social capital as a driver of local knowledge exchange: a social network analysis. Journal Knowledge Management Research y Practice, 12 (3), 276288 doi: $10.1057 / \mathrm{kmrp} .2014 .7$

Divisekera, S., y Nguyen, V. (2011). Determinants of innovation in tourism. Evidence from Australia. Tourism Management, 67, 157-167. doi: 10.1016/j.tourman.2018.01.010

Dredge, D. (2006). Networks, Conflict and Collaborative Communities. Journal of Sustainable Tourism, 14 (6), 562-581. doi: 10.2167/jost567.0 
Erkuş, H., y Eraydin, A. (2010). Environmental governance for sustainable tourism development: Collaborative networks and organization building in the Antalya tourism region. Tourism Management, 31 (1), 113-124. doi: 10.1016/j.tourman.2009.01.002

Faccin, K., Genari, D., y Macke, J. (2017). Interorganisational social capital and innovation: A multiple case study in wine producers networks in Serra Gaúcha. RAI Revista de Administração e Inovação, 14 (1), 52-66. doi: 10.1016/j.rai.2016.12.002

Farrell, H., Bosworth, G., y Newbery, R. (2010). Networking among Rural Tourism Entrepreneurs. WIT Transactions on Ecology and the Environment, 142, 715-723. doi: 10.2495/SW100641

Getz, D., y Petersen, T. (2005). Growth and Profit-Oriented Entrepreneurship among Family Business Owners in the Tourism and Hospitality. International Journal of Hospitality Management, 24 (2), 219-242.

Granovetter, M. (1985). Economic Action and Social Structure: The Problem of Embeddedness. American Journal of Sociology, 91, 481-510. doi: 10.1086/228311

Greenberg, Z., Farja, Y. y Gimmon, E. (2018). Embeddednes and growth of small business in rural regions. Journal of Rural Studies, 62, 174-182. doi: 10.1016/j.jrurstud.2018.07.016

Gunn, C. (1977). Industry pragmatism vs tourism planning. Leisure Sciences, 1 (1), 85-94. doi: 10.1080/01490407709512872

Hall, C. (2005). Tourism: Rethinking the social science of mobility. Harlow: Prentice-hall.

Hall, C. (2011). Policy learning and policy failure in sustainable tourism governance: From firstand second-order to thirrd-order change? Journal of Sustainable Tourism, 19 (4-5), 649-671. doi: 10.1080/09669582.2011.555555

Hall, C. M. (2011b). A typology of governance and its implications for tourism policy analysis. Journal of Sustainable Tourism, 19 (4-5), 437-457. doi: 10.1080/09669582.2011.570346

Hazra, S., Fletcher, J. y Wilkes, K. (2014). An evaluation of power relationsships among stakeholders in the tourism industrynetworks of Agra, India. Current Issues in Tourism, 20, 278-294. doi: 10.1080/13683500.2014.887662

Hite, J. (2005). Evolutionary Processes and Paths of Relationally Embedded Network Ties in Emerging Entrepreneurial Firms. Entrepreneurship Theory and Practice, 29 (1), 113144. doi: 10.1111/j.1540-6520.2005.00072.x

Hjalager, A. M. (2002). Repairing innovation defectiveness in tourism . Tourism Management, 23, 465-474. doi: 10.1016/S0261-5177(02)00013-4

Hjalager, A.-M. (2010). A review of innovation research in tourism. Tourism Management, 31 (1), 1-12. doi: 10.1016/j.tourman.2009.08.012

Huggins, R. (2000). The success and failure of policy-implanted inter-firm network iniciatives: motivations, processes and structure. Entrepreneurship y Regional Development, 12 (2), 111-135. doi: 10.1080/089856200283036 
Inkpen, A. y Tsang, E. (2005). Social Capital i, Networks, and Knowledge Tranfer . The Academy of Management Review, 30 (1), 146-165. doi: 10.5465/amr.2005.15281445

Jack, S., y Anderson, A. (2002). The effects of embeddedness on the entrepreneurial process. Journal of Business Venturing, 17, 467-587. doi: 10.5465/amr.2005.15281445

Jamal, T., y Getz, D. (1995). Collaboration Theory and Community Tourism Planning . Annals of Tourism Research, 22 (1), 186-204. doi: 10.1016/0160-7383(94)00067-3

Jenssen, J. y Koenig, H. (2002). The Effect of Social Networks of Resources Access and Business Start-ups. European Planning Studies, 10 (8), 1039-1046. doi: 10.1080/0965431022000031301

Johannisson, B., Ramírez, M. y Karlsson, G. (2002). The institutional embeddeness of local interfirm networks: a leverage for business creation. Estrepreneurship y Regional development: An International Journal, 14 (4), 297-315. doi: 10.1080/08985620210142020

Keeble, D., Lawson, C., Moore, B. y Wikilson, F. (1999). Collective Learning Processes, Networking, and "Insitutional Thickness" in the Cambridge Region . Regional Studies, 33 (4), 319-332.

Kelliher, F., Reinl, L., Johnson, T. y Joppe, M. (2018). The rol of trust in buiding rural tourism micro firm network engagement: A multi-case study. Tourism Management , 68, 1-12

Kimbu, A. N., y Ngoasong, M. (2016). Women as a vectors of social entrepreneurship. Annals of Tourism Research , 60, 63-79. doi: 10.1016/j.annals.2016.06.002

Kofler, I. y Marcher, A. (2018). Inter-organizational networks of smalll and medium sized enterprises (SME) in the field of innovation: a case study of South Tyrol. Journal of Small Business y Entrepreneurship, 30 (1), 9-25.

Kofler, I., Marcher, A., Volgger, M. y Pechlaner, H. (2018). The special characteristics of tourism innovation networks: The case of the Regional Innovation in South Tyrol. Journal of Hospitality and Tourism Management, 37, 68-75. doi: 10.1080/08276331.2017.1401202

Laschewski, L., Phillipson, J., y Gorton, M. (2002). The Facilitaction ans Formalisation of Small Business Networks: Evidence from the Noth East of England. Environment and Planning C: Politics and Space, 20, 375-391. doi: 10.1068/c0066a

Lemmetyinen, A. y Frank, G. (2009). The key capabilities requiered for managing tourism business networks. Tourism Management, 30 (1), 31-40. doi: 10.1016/j.tourman.2008.04.005

Martínez, Á., Elche, D. y García, P. (2019). From diversity of interorganizational relationships to radical innovation intourism destination: The role of knowledge exploration. Journal of Destination Marketing and Management, 11, 80-88. doi: 10.1016/j.jdmm.2018.12.002

McCabe, S., Sharples, M. y Foster, C. (2012). Stakeholder engagement in the design of scenarios of technology-enhanced tourism services. Tourism Management Perspectives , 4, 36-44. 
McComb, E. y Boyd, S. (2016). Stakeholders collaboration: A means to the success of rural tourism destinations?. A critical evaluation of the existence of stakeholder collaboration within the Mournes, Northern Ireland. Tourism and Hospitality Research, 0 (0), 1-12.

Melián, A., y García, J. M. (2003). Competitive potencial of tourism in destinations. Annals of Tourism Research, 30 (3), 720-740. doi: 10.1016/S0160-7383(03)00047-1

Mu, J., Peng, G. y Love, E. (2008). Interfirm networks, social capital, and knoledge flow. Journal of Knowledge Management, 12 (4), 86-100. doi: 10.1108/13673270810884273

Narváez, M. y Fernádez, G.( 2013). Redes de cooperación empresarial: Relaciones e interacciones para promover desarrollo turístico local. Revista Venezolana de Gerencia, 18 (61), 121-137. doi: 10.31876/revista.v18i61.11007

Novelli, M., Schmitz, B. y Spencer, T. (2006). Networks, clusters and innovation in tourism: A UK experience. Tourism Management, 27, 1141-1152. doi: 10.1016/j.tourman.2005.11.011

Polanyi, K. (1992). La Gran Tranformación. Madrid, España: Ediciones de La Piqueta.

Powell, W., y Grodal, S. (2006). Networks of Innovators. En J. Fagerberg, D. Mowery, y R. Nelson, The Oxford Handbook of Innovation. Oxford: Oxford University Press. doi: 10.1093/oxfordhb/9780199286805.003.0003

Proctor, A., Donaldson, A., y Phillipson, J. (2012). Field Expertise in Rural Land Management. Environement and Planning A: Economy and Space, 44, 1696-1711. doi: $10.1068 / \mathrm{a} 44352$

Quaranta, G., Citro, E., y Salvia, R. (2016). Economic and Social Sustainable Synergies to Promote Innovations in Rural Tourism and Local Development. Sustainability, 8 (7), 115. doi: $10.3390 /$ su 8070668

Ramayah, T., Lee, J. C. y In, J. (2011). Network collaboration and performance in the tourism sector. Service Business , 5 (411), 42-62. doi: 10.1007/s11628-011-0120-z

Saxena, G. (2005). Relationships, networks and the learning regions: case evidence from the Peak District National Park. Tourism Management, 26, 277-289. doi: 10.1016/j.tourman.2003.11.013

Scott, W. (2001). Institutions and Organizations. Ideas, Interests and Identities. California: SAGE

Seelos, C., Mair, J., Battilana, J. y Dacin, T. (2015). The embeddedness of social entrepreneurship understanding variation across local communities. Communities and Organizations, 33, 333-363. doi: 10.1108/S0733-558X(2011)0000033013

Shockley, P., Ellis, K., y Winograd, G. (2000). Organizational trust: What it means, why it matters. Organization Development Journal, 18 (4), 35-48.

Skokic, V., Lynch, P., y Morrison, A. (2019). Veza: An informal network of tourism entrepreneurs. Annals of Tourism Research, 77, 26- 37. doi: 10.1016/j.annals.2019.05.004 
Soisalo, T., y Lindroth, K. (2006). Regional tourism co-coperation in progress. En L. Lazzeretti, y C. Petrillo, Tourism local systems and networking (págs. 187-196). Amsterdam: Elsevier.

Sorensen, F. (2007). The Geographies of Social Networks and Innovation in Tourism. Tourism Geographies: An International Journal of Tourism Space, Place and Environment, 9 (1), 22-48. doi: 10.1080/14616680601092857

Stoddarta, M., Catano, G., Ramos, H., Vodden, K., Lowery, B. y Butters, L. (2019). Collaboration gaps and regional tourism networks in rural coastal communities. Journal of Sustainable Tourism, 28 (4), 625-645. doi: 10.1080/09669582.2019.1694526

Thomas, H. y Thomas, R. (1999). The implications for tourism of shifts in British local governance. Progress in Tourism and Hospitality Research , 4 (4), 295-306.

Tolstad, H. (2014). Development of rural-tourism experiences through networking: An example from Gudbrandsdalen. Norsk Geografisk Tidsskrift - Norwegian Journal of Geography , 68 (2), 111-120. doi: 10.1080/00291951.2014.894561

Tregear, A. y Cooper, S. (2016). Embeddedness, social capital and learning in rural areas: the case of producer cooperatives. Journal of Rural Studies, 44, 101-110. doi: 10.1016/j.jrurstud.2016.01.011

Trist, E. (1977). A Concept of Organizatinal Ecology. Australian Journal of Management , 2 (2), 161-175. doi: 10.1177/031289627700200205

Uzzi, B. (2000). the Source and Consequences of Embeddeness and the Economic Perfomance of Organizations: The Network Effect. American Sociological Review , 61 (4), 674-698. doi: $10.2307 / 2096399$

van der Zee, E., y Vanneste, D. (2015). Tourism networks unravelled; a review of the literature on networks in tourism management studies. Tourism Management Perspectives, 15, 46-56. doi: 10.1016/j.tmp.2015.03.006

Wang, Y., y Fesenmaier, D. (2007). Collaborative destination marketing: A case study of Elkhart county, Indiana. Tourism Management, 28 (3), 863-875. doi: 10.1016/j.tourman.2006.02.007

Weidenfeld, A. (2013). Tourism ans cross border regional innovation. Annals of Tourism Research, 42, 191-213. doi: 10.1016/j.annals.2013.01.003

Wigren, C., Korsgaard, S., Brindin, E., Hellerstedt, K., Agnete, G., y Jorunn, G. (2019). Entrepreneurship and embeddedness: dynamic, processual and multi-layered perspectives . Entrepreneurship y Regional Development, 31 (9-10), 1011-1015. doi: 10.1080/08985626.2019.1656868

Ying-Hueih, C., Tzu-Pei, L. y David, Y. (2014). How to facilitate inter-organizational knowledge sharing: The impact of trust. Information y Management, 51 (5), 568-578. doi: 10.1016/j.im.2014.03.007

Zhang, J. (2010). The problems of using social networks in entrepreneurial resource acquisition. International Small Busines Journal: Researching Entrepreneurship , 28 (4), 338-361. doi: 10.1177/0266242610363524 


\section{CONTRIBUCIONES DE LOS AUTORES:}

Autor 1: concepción original del trabajo, análisis, adquisión e intepretación de los datos y redacción y revisión crítica del texto.

Autor 2: redacción y revisión crítica del texto, aprobación sinal de la revisión a publicar.

Autor 3: redacción y revisión crítica del texto, aprobación sinal de la revisión a publicar. 
ANEXO

Tabla de co-ocurrencias entre códigos

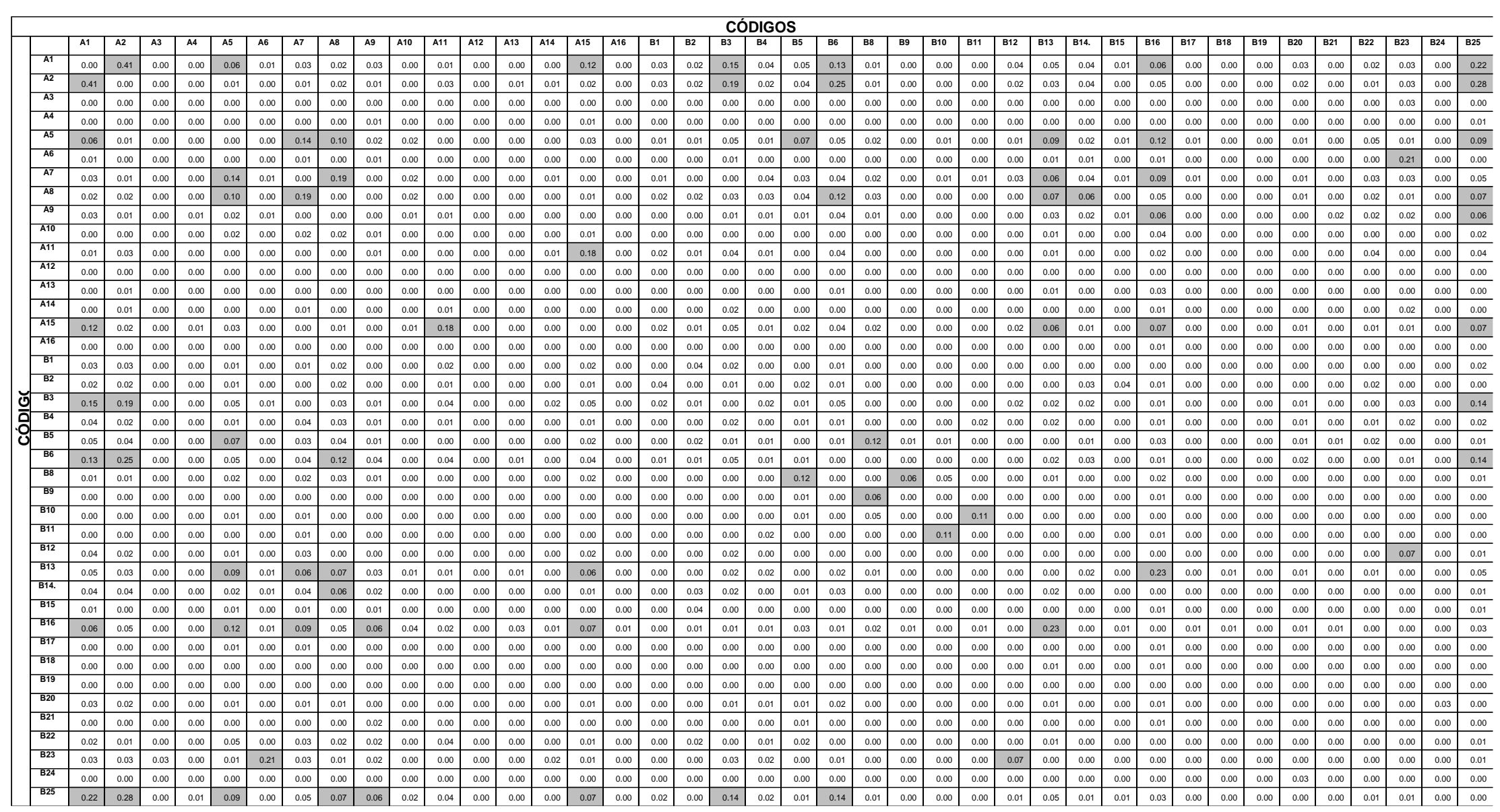

Fuente: Elaboración propia a partir del análisis de los datos 